\title{
Role of lymphadenectomy in disease-free and overall survival on low risk endometrium cancer patients
}

\author{
Can Turkler ${ }^{1,2}$, Mehmet Kulhan ${ }^{1,2}$, Nur Gozde Kulhan ${ }^{1,2}$, Nahit Ata ${ }^{1,2}$, \\ Muzaffer Sanci², Mehmet Ozeren² \\ ${ }^{1}$ Erzincan University Medical Faculty, Gynaecology and Obstetrics Department, Erzincan, Turkey \\ 2izmir Tepecik Training and Research Hospital, Gynaecology and Obstetrics Department, Izmir, Turkey
}

\begin{abstract}
Objectives: Evaluation of the effect of lymphadenectomy in disease-free and overall survival on the low risk corpus cancer. Material and methods: Between 1994 and 2012, a total of 257 patients with endometrioid type, grade 1 or 2, myometrial invasion $<1 / 2$, no intraoperative evidence of macroscopic extrauterine spread was treated surgically. Pelvic lymphadenectomy was performed in 184 cases, and not performed in 73 cases.

Results: There was no difference between two groups about tumor sizes. Also lymphovascular space invasion and histologic grade of two groups were similar. Omission of LA did not worsen DFS and OS in early stage low risk corpus cancer.

Conclusions: Patients who have low risk corpus cancer, can be treated optimally with hysterectomy only.

Key words: endometrial cancer, low risk, lymphadenectomy
\end{abstract}

Ginekologia Polska 2018; 89, 6: 311-315

\section{INTRODUCTION}

Endometrial cancer is the most common malignancy of the female genital tract, in many developed countries [1]. 10,920 women lost their life because of endometrial cancer, in the United States in 2017 [2].

When $80 \%$ of patients are caught by a clinician, tumor confined to the corpus uteri (stage 1). Five-year survival rate of localized cancers $95.5 \%$ at the Surveillance Epidemiology and End Results (SEER) data [3]. The International Federation of Gynecology and Obstetrics (FIGO) recommended in 1988, standard staging surgery for endometrial cancer is total extrafascial hysterectomy, bilateral salpingo-oophorectomy and pelvic-paraaortic lymphadenectomy (PPLA). In 2009, last revised surgical staging system for endometrial cancer, which includes lymphadenectomy, was reported by the FIGO $[4,5]$. The American College of Obstetrics and Gynecologists (ACOG) recommended to perform systematic LA rather than nodal sampling [6].

Hidaka et al, did not suggest systemic LA for low-risk corpus cancer (endometrioid type, grade 1 or 2, myome- trial invasion $<1 / 2$, no intraoperative evidence of macroscopic extrauterine spread) because there is no difference between DFS and OS [7]. Some retrospective studies have recommended that a therapeutic benefit is associated with lymphadenectomy (LA) in early-stage endometrial cancer $[8,9]$. There is a controversy about benefit of PLA in patients with early stage endometrial cancer $[10,11]$.

\section{Objectives}

In this study we investigated whether LA was necessary or not in early stage endometrial cancer. The primary outcome was to see the role of LA in DFS and OS on low risk endometrial cancer patients. The second outcomes were to learn demographic specialities, comorbid conditions, pre-op Cancer Antigen-125 (CA-125) levels and end-organ metastasis.

\section{MATERIAL AND METHODS}

Two hundred fifty seven patients who were operated due to endometrial carcinoma which have endometrioid type, FIGO stage 1a, grade 1 or 2 histology at the Izmir 
Tepecik Research and Teaching Hospital, between 1994 and 2012, were included in this study. We eliminated patients who had preoperative therapy, histologic grade 3, other histologic types and other FIGO stages from the study. Clinical, pathological and surgical data were collected. All patients were evaluated for OS and DFS. The following clinical data were collected from patient medical, surgical, pathological reports: demographic characteristics, presenting symptoms, serum CA-125 level, date and type of the surgical procedure, presence or absence of residual tumor after surgery, number of excised and positive lymph nodes, presence or absence of ascites, tumor pathological characteristics (grade and size), date of recurrence, treatment after recurrence, date of the last medical examination and date of death.

Primarily cases were divided into two groups, underwent lymph node dissection (LND) and non-lymph node dissection (non-LND). The patients were classified as 'non-Lymph node dissection' if only a total hysterectomy with unilateral or bilateral salpingo-oophorectomy with or without omentectomy was performed. Complete staging was defined as pelvic washing, omentectomy, bilateral pelvic and para-aortic lymph node dissection with bilateral salpingo-oophorectomy and total abdominal hysterectomy. Patients who underwent complete staging were defined as 'Lymph node dissection'. 184 patients underwent lymph node dissection, and 73 patients did not. The decision whether or not to perform lymph node dissection was made according to the results of intraoperative pelvic examination findings and frozen section results.

The patients returned for a follow-up every three months for the first 2 years, every 6 months for the next 3 years, and annually, thereafter. Computed tomography or magnetic resonance imaging was performed annually. The revised
2009 FIGO staging system was used. All groups analyzed demographic data, survival periods, recurrence and metastasis. Comorbid conditions, pre-op CA-125 levels, tumor diameters were other findings.

The survival analysis was based on the Kaplan-Meier method, and the results were compared using the log-rank test. DFS was defined as the time from the date of the primary surgery to detection of recurrence or the latest observation. OS was defined as the time from the date of primary surgery to death or the latest observation. The chi-square test and Student's $t$ test for unpaired data were used for statistical analyses. Cox regression analysis was used to determine factors affecting the survival, and the results are presented as Hazard Ratios (HRs), with 95\% Confidence Intervals (Cls). All statistical analyses were performed using Statistical Package for the Social Sciences (SPSS ver.11.5). The $p$-value of $<0.05$ was considered as statistically significant.

\section{RESULTS}

Ninety eight patients underwent PLA, 86 patients underwent PPLA in this study. There was no lymph node spreading and positive peritoneal cytology at LND group.

The age range in the LND group was 34-77 (mean age, $53.9 \pm 7.9$ years), whereas in the non-LND group was $40-$ -74 (mean age, $54.8 \pm 7.4$ years). Comorbid disease had seen much more at non-LND cases, than LND cases ( $p<0.05$ ). Demographic data is summarized in Table 1.

When the postoperative pathology specimens of two groups had been analyzed, we saw there were no difference about primary tumor diameter. Also there were no difference about histologic grade and LVSI ratio. Preoperative CA-125 levels of cases at non-LND group less than LND

\begin{tabular}{|c|c|c|c|c|}
\hline & & $\begin{array}{l}\text { Not undergone lymphadenectomy } \\
\qquad \begin{array}{c}(n=73) \text { mean } \pm \text { SD } \\
n-\%\end{array}\end{array}$ & $\begin{array}{l}\text { Undergone lymphadenectomy } \\
\qquad \begin{array}{c}(n=184) \text { mean } \pm S D \\
n-\%\end{array}\end{array}$ & $\mathbf{p}$ \\
\hline \multicolumn{2}{|l|}{ Age } & $54.8 \pm 7.4$ & $53.9 \pm 7.9$ & 0.391 \\
\hline \multicolumn{2}{|c|}{ First menstruation age } & $13.3 \pm 1.1$ & $13.1 \pm 1.0$ & 0.149 \\
\hline \multicolumn{2}{|c|}{ Last menstruation age ${ }^{*}$} & $48.7 \pm 3.5(n: 51)$ & $48.5 \pm 4.0(n: 136)$ & 0.820 \\
\hline \multicolumn{2}{|l|}{ Parity } & $2.7 \pm 1.1$ & $3.0 \pm 1.5$ & 0.338 \\
\hline \multirow{2}{*}{ Menopause } & no & $22(\% 30.1)$ & $48(\% 26.1)$ & \\
\hline & yes & $51(\%$ 69.9) & $136(\% 73.9)$ & \\
\hline \multirow{2}{*}{ Comorbid disease } & no & $33(\% 45.2)$ & 109 (\% 59.2) & \multirow{2}{*}{0.041} \\
\hline & yes & $40(\% 54.8)$ & $75(\%$ 40.8) & \\
\hline \multicolumn{2}{|l|}{ HT } & $22(\% 30.1)$ & $38(\%$ 20.6) & \\
\hline \multicolumn{2}{|l|}{ DM } & $6(\% 8.2)$ & $14(\% 7.6)$ & \\
\hline \multicolumn{2}{|l|}{$\mathrm{HT}+\mathrm{DM}$} & $11(\%$ 15) & (\% 9.7) & \\
\hline
\end{tabular}

* - Mean age of cases at menopause; DM — Diabetes Mellitus; HT — Hypertension 
Table 2. Preoperative CA-125 levels and tumor diameters, histologic characteristics at postoperative specimens

\begin{tabular}{|c|c|c|c|c|}
\hline & & $\begin{array}{l}\text { Not undergone lymphadenectomy } \\
\qquad \begin{array}{c}(n=73) \text { mean } \pm S D \\
n-\%\end{array}\end{array}$ & $\begin{array}{l}\text { Undergone lymphadenectomy } \\
\qquad \begin{array}{c}(n=184) \text { mean } \pm \text { SD } \\
N-\%\end{array}\end{array}$ & $\mathbf{p}$ \\
\hline \multicolumn{2}{|l|}{ Tumor diameter } & $2.45 \pm 1.68 \mathrm{~cm}$ & $2.37 \pm 1.38 \mathrm{~cm}$ & 0.846 \\
\hline \multicolumn{2}{|l|}{ CA-125 level } & $10.9 \pm 9.0$ & $15.6 \pm 31.2$ & 0.025 \\
\hline \multirow{2}{*}{ LVSI } & no & $71(\% 97.3)$ & 180 (\% 97.8) & \multirow{2}{*}{1,000} \\
\hline & yes & $2(\% 2.7)$ & $4(\% 2.2)$ & \\
\hline \multirow{2}{*}{ Histologic grade } & 1 & $51(\%$ 69.9) & 125 (\% 67.9) & \multirow{2}{*}{0.764} \\
\hline & 2 & $22(\% 30.1)$ & $59(\% 32.1)$ & \\
\hline Metastasis & & $1(\% 1.4)$ & $8(\% 4.3)$ & 0.242 \\
\hline Exitus & & $0(\% 0)$ & $2(\% 1.1)$ & 1,000 \\
\hline
\end{tabular}

group ( $p<0.05)$. Level range of preoperative CA-125 levels in non-LND and LND group were 3-64 U/mL, 2-410 U/mL, respectively. Clinicopathological characteristics of the patients are summarized in Table 2.

Eight cases had end-organ metastasis at LND group, 2 cases had lung metastasis (postoperative $40^{\text {th }}$ and $60^{\text {th }}$ month), 2 cases had liver metastasis (postoperative $8^{\text {th }}$ and $17^{\text {th }}$ month), 1 case had colon metastasis (postoperative $48^{\text {th }}$ month), 1 case had bone metastasis (postoperative $7^{\text {th }}$ month), 1 case had rectum metastasis (postoperative $84^{\text {th }}$ month), 1 case had paracolic peritoneal metastasis (postoperative $9^{\text {th }}$ month). One patient had liver metastasis (postoperative $30^{\text {th }}$ month) at non- LND group.

Two patients died at LND group, the first one, who had bone metastasis, died at postoperative $12^{\text {th }}$ month, second patient died in the $21^{\text {st }}$ month who had liver metastasis at postoperative $17^{\text {th }}$ month. There was no local recurrence both of two groups, and no difference about metastasis and exitus statistics. Also we did not see any statistical difference between two groups about DFS and OS.

Metastatic and non-metastatic patients in LND group analyzed at Table 3. There were no statistical differences about tumor diameter, LVSI and CA-125 levels between metastatic and non-metastatic patients ( $p>0.05)$. At metastatic group, histologic grade and exitus rate were upper than non-metastatic group $(p<0.05)$.

\section{DISCUSSION}

FIGO recommended a systemic surgical staging system for corpus cancer, in 1988 [12]. After this recommendation, controversy has become the role of the addition of LA to surgical procedures [13]. Multiple retrospective studies have shown that a low-risk endometrial cancer patients had a small overall risk of lymph node involvement [14-16]. In our study, LVSI rate was $2.7 \%$ at non-LND group, and 2.2\% at LND group. Also there was no lymph node spreading, both two study groups.

Trimble et al. demonstrated that the 5 -year relative survival for 6363 women with stage I endometrial cancer who did not undergo lymph node sampling was $98 \%$, compared to $96 \%$ for 2831 women who underwent lymph node sampling. It means that lymph node sampling did not appear to convey survival benefit [17]. When we analyzed our cases, DFS was $88.8 \pm 43.2$ months, OS was $91.0 \pm 43.5$ months in LND group. On the other hand, DFS was $97.9 \pm 48.7$ months, OS was $98.8 \pm 48.1$ months in non-LND group. There were no

Table 3. CA-125 levels, tumor diameters, histologic grades and exitus numbers of undergone lymphadenectomy patients

\begin{tabular}{|c|c|c|c|c|}
\hline \multicolumn{2}{|c|}{$\begin{array}{l}\text { Undergone lymphadenectomy } \\
\text { patients }(n=184)\end{array}$} & $\begin{array}{c}\text { Non-Metastatic patients } \\
\text { Ort. } \pm \text { S.S./n }-\%\end{array}$ & $\begin{array}{c}\text { Metastatic patients } \\
\text { Ort. } \pm \text { S.S./n - } \%\end{array}$ & $\mathbf{p}$ \\
\hline \multicolumn{2}{|l|}{ Tumor diameter } & $2.36 \pm 1.39 \mathrm{~cm}$ & $2.38 \pm 1.22 \mathrm{~cm}$ & 0.940 \\
\hline \multicolumn{2}{|l|}{ CA-125 level } & $13.3 \pm 10.9$ & $66.5 \pm 139.2$ & 0.075 \\
\hline \multirow{2}{*}{ Exitus } & no & $176(\% 100)$ & $6(\% 75)$ & \multirow{2}{*}{0.002} \\
\hline & yes & $0(\% 0)$ & $2(\% 25)$ & \\
\hline \multirow{2}{*}{ LVSI } & no & $172(\% 97.7)$ & $8(\% 100)$ & \multirow{2}{*}{1,000} \\
\hline & yes & $4(\% 2.3)$ & $0(\% 0)$ & \\
\hline \multirow{2}{*}{ Histologic grade } & 1 & 123 (\% 69.9) & $2(\% 25)$ & \multirow{2}{*}{0.008} \\
\hline & 2 & $53(\%$ 30.1) & $6(\% 75)$ & \\
\hline
\end{tabular}




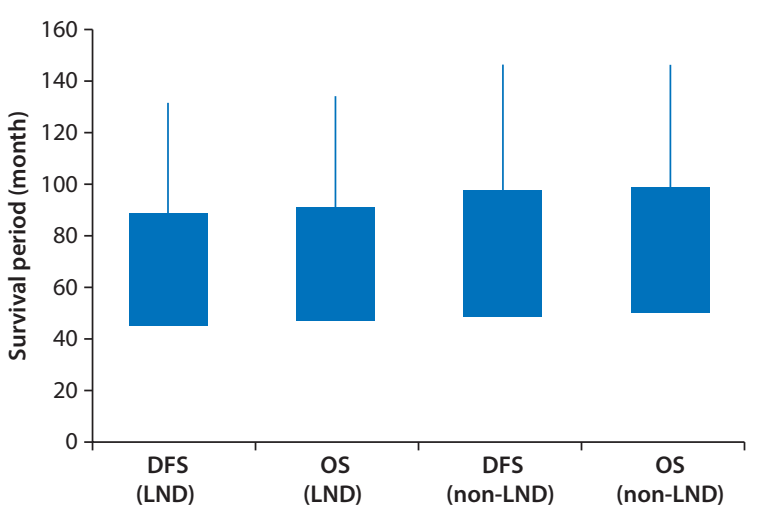

Figure 1. Survival graphic of our cases

significant differences between two groups about survival, as shown in Figure 1 ( $p=0.828$ ).

Schink et al. presented a $4 \%$ risk of lymph node involvement when tumor size was $<2 \mathrm{~cm}$, compared with $15 \%$ if tumors were $>2 \mathrm{~cm}$ [18]. Primary tumor diameter has been described as a predictor of lymph node invasion in endometrial cancer [19]. Mariani et al. indicated that a low-risk group could be defined as patients with: (i) endometrioid subtype; (ii) myometrial invasion of $50 \%$ or less; (iii) histological grade 1-2 and (iv) no intraoperative evidence of macroscopic disease. In 2004, they revised the criteria (Mayo criteria) and tumor size (diameter of $\leq 2 \mathrm{~cm}$ ) was newly introduced [20]. In this study, mean tumor sizes were $2.37 \mathrm{~cm}$ and $2.45 \mathrm{~cm}$ in the LND group and non-LND group, respectively. This finding, was different from literature. The importance of tumor size about lymph node spreading, should be confirmed in future prospective large-scale randomized clinical trials in the low risk corpus cancer.

Most of the endometrium cancer patients have comorbid diseases such as obesity, diabetes mellitus, hypertension and metabolic syndrome [21]. In the present study, most common comorbid diseases were hypertension and diabetes mellitus, as shown in Table 1.

There were no statistical difference, about end-organ metastasis between two groups $(p=0.242)$. We have not seen any vaginal recurrence in this study.

Different preoperative predictor types were studied to detect lymph node involvement to prevent unnecessary lymph node dissection. CA 125 and different imaging modalities were found as useful predictors for more advanced disease [22]. MRI data and serum CA-125 levels were used at the preoperative screening of lymph node metastasis in low risk endometrial cancer. Serum CA-125 levels greater than $35 \mathrm{U} / \mathrm{mL}$ is a risk factor [23]. In this study, interval of preoperative CA-125 levels 3-64 U/mL at non-LND group, 2-410 U/mL at LND group. When we analyzed Table 3, preoperative CA-125 levels upper than $35 \mathrm{U} / \mathrm{mL}$ at metastatic patients, and less than $35 \mathrm{U} / \mathrm{mL}$ at non-metastatic patients. Therefore, the possibility of end-organ metastasis increases in patients with high pre-operative CA-125 values, making lymphadenectomy seem reasonable. If lymphadenectomy is performed, CA-125 and other risk factors must be considered.

This study has several limitations. First of all, this is a cross-sectional study with a small sample size. Secondly, it is a retrospective study. The third disadvantage was that surgeries performed by different surgeons.

\section{CONCLUSIONS}

As shown in this study, women with low risk endometrial cancer, as defined by the Mayo criteria, have a low rate of lymph node metastasis. Also, there was no survival benefit of LA in DFS and OS on low risk endometrial cancer patients, same with literature $(p=0.828)$. This results should be confirmed in future, prospective large-scale randomized clinical trials.

\section{REFERENCES}

1. Kulhan M, Kulhan G, Nayki U, et al. Assessment of clinicopathological features, evaluation of treatment, and prognosis of clear cell and serous papillary endometrial carcinoma. Ginekol Pol. 2016; 87(8): 570-574, doi: 10.5603/GP.2016.0046, indexed in Pubmed: 27629131.

2. Siegel RL, Miller KD, Jemal A. Cancer Statistics, 2017. CA Cancer J Clin. 2017; 67(1): 7-30, doi: 10.3322/caac.21387, indexed in Pubmed: 28055103.

3. Arem $\mathrm{H}$, Irwin $\mathrm{ML}$, Zhou Y, et al Physical activity and endometrial cancer in a population-based case-control study. Cancer Causes Control. 2011; 22(2): 219-226, doi: 10.1007/s10552-010-9689-0, indexed in Pubmed: 21110224

4. Shepherd JH. Revised FIGO staging for gynaecological cancer. Br J Obstet Gynaecol. 1989; 96(8): 889-892, doi: 10.1111/j.1471-0528.1989. tb03341.x, indexed in Pubmed: 2775686.

5. Pecorelli S. Revised FIGO staging for carcinoma of the vulva, cervix, and endometrium. Int J Gynaecol Obstet. 2009; 105(2): 103-104, doi: 10.1016/j.ijgo.2009.02.012, indexed in Pubmed: 19367689.

6. Fotopoulou C, Savvatis K, Kraetschell R, et al. Systematic pelvic and aortic lymphadenectomy in intermediate and high-risk endometrial cancer: lymph-node mapping and identification of predictive factors for lymph-node status. Eur J Obstet Gynecol Reprod Biol. 2010; 149(2): 199-203, doi: 10.1016/j.ejogrb.2009.12.021, indexed in Pubmed: 20096987.

7. Hidaka T, Nakashima A, Shima T, et al. Systemic lymphadenectomy cannot be recommended for low-risk corpus cancer. Obstet Gynecol Int. 2010; 2010: 490219, doi: 10.1155/2010/490219, indexed in Pubmed: 20168975.

8. Cragun JM, Havrilesky LJ, Calingaert B, et al. Retrospective analysis of selective lymphadenectomy in apparent early-stage endometrial cancer. J Clin Oncol. 2005; 23(16): 3668-3675, doi: 10.1200/JCO.2005.04.144, indexed in Pubmed: 15738538.

9. Zhang $\mathrm{H}$, Jia L, Zhang $\mathrm{Q}$, et al. Therapeutic role of systematic retroperitoneal lymphadenectomy in endometrial cancer. Bull Cancer. 2012; 99(2): E10-E17, doi: 10.1684/bdc.2011.1536, indexed in Pubmed: 22266042.

10. Benedetti Panici P, Basile S, Maneschi F, et al. Systematic pelvic lymphadenectomy vs. no lymphadenectomy in early-stage endometrial carcinoma: randomized clinical trial. J Natl Cancer Inst. 2008; 100(23): 1707-1716, doi: 10.1093/jnci/djn397, indexed in Pubmed: 19033573.

11. Kitchener $\mathrm{H}$, Swart AMC, Qian $\mathrm{O}$, et al. ASTEC study group. Efficacy of systematic pelvic lymphadenectomy in endometrial cancer (MRC ASTEC trial): a randomised study. Lancet. 2009; 373(9658): 125-136, doi: 10.1016/S0140-6736(08)61766-3, indexed in Pubmed: 19070889.

12. FIGO stages 1988 revision. Gynecol Oncol. 1989; 35: 125-126.

13. Mariani A, Webb MJ, Keeney GL, et al. Low-risk corpus cancer: is lymphadenectomy or radiotherapy necessary? Am J Obstet Gynecol. 2000; 182(6): 1506-1519, doi: 10.1067/mob.2000.107335, indexed in Pubmed: 10871473. 
14. Convery PA, Cantrell LA, Di Santo N, et al. Retrospective review of an intraoperative algorithm to predict lymph node metastasis in low-grade endometrial adenocarcinoma. Gynecol Oncol. 2011; 123(1): 65-70, doi: 10.1016/j.ygyno.2011.06.025, indexed in Pubmed: 21742369.

15. Milam MR, Java J, Walker JL, et al. Gynecologic Oncology Group. Nodal metastasis risk in endometrioid endometrial cancer. Obstet Gynecol. 2012; 119 (2 Pt 1): 286-292, doi: 10.1097/AOG.0b013e318240de51, indexed in Pubmed: 22270280

16. Shah $C$, Johnson EB, Everett $E$, et al. Does size matter? Tumor size and morphology as predictors of nodal status and recurrence in endometrial cancer. Gynecol Oncol. 2005; 99(3): 564-570, doi: 10.1016/j. ygyno.2005.06.011, indexed in Pubmed: 16246402.

17. Trimble EL, Kosary C, Park RC. Lymph node sampling and survival in endometrial cancer. Gynecol Oncol. 1998; 71(3): 340-343, doi: 10.1006/gyno.1998.5254, indexed in Pubmed: 9887227.

18. Schink JC, Rademaker AW, Miller DS, et al. Tumor size in endometrial cancer. Cancer. 1991; 67(11): 2791-2794, doi: 10.1002/1097-0142(19910601)67:11<2791::aid-cncr2820671113>3.0. co;2-s, indexed in Pubmed: 2025843.
19. Kamura $\mathrm{T}$, Yahata $\mathrm{H}$, Shigematsu $\mathrm{T}$, et al. Predicting pelvic lymph node metastasis in endometrial carcinoma. Gynecol Oncol. 1999; 72(3): 387-391, doi: 10.1006/gyno.1998.5288, indexed in Pubmed: 10053111.

20. Mariani A, Dowdy SC, Keeney GL, et al. High-risk endometrial cancer subgroups: candidates for target-based adjuvant therapy. Gynecol Oncol. 2004; 95(1): 120-126, doi: 10.1016/j.ygyno.2004.06.042, indexed in Pubmed: 15385120

21. Mariani A, Dowdy SC, Cliby WA, et al. Prospective assessment of lymphatic dissemination in endometrial cancer: a paradigm shift in surgical staging. Gynecol Oncol. 2008; 109(1): 11-18, doi: 10.1016/j. ygyno.2008.01.023, indexed in Pubmed: 18304622.

22. Kang S, Todo Y, Watari H. Risk assessment of lymph node metastasis before surgery in endometrial cancer: do we need a clinical trial for low-risk patients? J Obstet Gynaecol Res. 2014; 40(2): 322-326, doi: 10.1111/jog.12281, indexed in Pubmed: 24428370.

23. Kang $\mathrm{S}$, Kang WD, Chung $\mathrm{HH}$, et al. Preoperative identification of a low-risk group for lymph node metastasis in endometrial cancer: a Korean gynecologic oncology group study. J Clin Oncol. 2012; 30(12): 1329-1334, doi: 10.1200/JCO.2011.38.2416, indexed in Pubmed: 22412131. 